\title{
Review of: "Strong SARS-CoV-2 N-specific CD8+ T immunity induced by engineered extracellular vesicles associates with protection from lethal infection in mice"
}

\section{Riccardo Gavioli ${ }^{1}$}

1 University of Ferrara

Potential competing interests: The author(s) declared that no potential competing interests exist.

New vaccine strategies against SARS-CoV-2 are needed to overcome some limitations of anti-SARS-CoV-2 vaccines currently used. The study by Ferrantelli et al. may contribute to the identification of new vaccine approaches also able to induce T cell responses against viral antigens. The design of the study is well performed, clearly presented and the interpretation of the data is coherent with the obtained results. I do not have any suggestion to further improve the manuscript. 